\title{
Electromagnetic Hadron Form Factors and Higher Fock Components
}

\author{
J. P. B. C. de Melo ${ }^{\text {a }}$, T. Frederico ${ }^{b}$, E. Pace and S. Pisano ${ }^{c}$, G. Salmè ${ }^{d}$ \\ ${ }^{a}$ Centro de Ciências Exatas e Tecnológicas, Universidade Cruzeiro do Sul, 08060-070, \\ and Instituto de Física Teórica, Universidade Estadual Paulista 01405-900, São Paulo, \\ Brazil \\ ${ }^{\text {b}}$ Dep. de Física, Instituto Tecnológico da Aeronáutica, Centro Técnico Aeroespacial, \\ 12.228-900 São José dos Campos, São Paulo, Brazil \\ "Dipartimento di Fisica, Università di Roma "Tor Vergata" and Istituto Nazionale di \\ Fisica Nucleare, Sezione Tor Vergata, Via della Ricerca Scientifica 1, I-00133 Roma, Italy \\ ${ }^{\text {d} I s t i t u t o ~ N a z i o n a l e ~ d i ~ F i s i c a ~ N u c l e a r e, ~ S e z i o n e ~ d i ~ R o m a ~}$ \\ P.le A. Moro 2, I-00185 Roma, Italy
}

Investigation of the spacelike and timelike electromagnetic form factors of hadrons, within a relativistic microscopical model characterized by a small set of hypothesis, could shed light on the components of hadron states beyond the valence one. Our relativistic approach has been successfully applied first to the pion and then the extension to the nucleon has been undertaken. The pion case is shortly reviewed as an illustrative example for introducing the main ingredients of our approach, and preliminary results for the nucleon in the spacelike range $-10(\mathrm{GeV} / \mathrm{c})^{2} \leq q^{2} \leq 0$ are evaluated.

\section{INTRODUCTION}

The global analysis of the electromagnetic (EM) form factors in both the spacelike (SL) region and the timelike (TL) one, within an approach based on the Light-Front language [1, 2], allows one to investigate in great detail the issue of non-valence components of both hadron and photon wave functions. In Refs. [ [3, 4], the pion EM form factor, in the range of momentum transfer $-10(\mathrm{GeV} / \mathrm{c})^{2} \leq q^{2} \leq 10(\mathrm{GeV} / \mathrm{c})^{2}$, has been analyzed within our relativistic approach, based on: i) a microscopic Vector Meson Dominance (VMD) model for the dressed vertex of the photon, and ii) a simple parametrization for the emission/absorption of a pion by a quark. Indeed, the pion represents a suitable introduction for illustrating the main features of our approach [3, 4].

Light-front dynamics (LFD) approaches [2] yield a unique possibility to study the hadronic state, in both the valence and the nonvalence sectors, since within LFD a Fock series for the state of a fermionic system, like

$$
\begin{aligned}
\mid \text { meson }\rangle & =|q \bar{q}\rangle+|q \bar{q} q \bar{q}\rangle+|q \bar{q} g\rangle \ldots . \\
\mid \text { baryon }\rangle & =|q q q\rangle+|q q q q \bar{q}\rangle+|q q q g\rangle \ldots .
\end{aligned}
$$


is a meaningful tool for investigating hadronic properties. It is worth noting that the so-called zero modes allow one to produce the breaking of the chiral symmetry through a different mechanism from the standard one, so that the LF vacuum acquires a more simple structure. Moreover, coupling the study of the EM form factors in the TL region with the analysis in the SL one, yields the possibility to address the vast phenomenology of hadronic resonances, e.g., the vector meson (VM) propagation, and consequently to impose strong constraints on dynamical models devised for a microscopical description of hadrons. Finally, one can obtain insight into the two-body currents associated to the $q \bar{q}$ pair production, that plays a very relevant role in a reference frame where $q^{+} \neq 0$ (see, e.g. [5]), namely the frame mandatory for carrying on our global analysis of the EM form factors.

\section{The Mandelstam formula for the EM current}

Our guidance for constructing a microscopical model that embeds, at large extent, the advantages of a LF approach is given by the Mandelstam formula [6] for the matrix elements of the EM current of a system of interacting constituents. In a global investigation of SL and TL regions one needs to change frame, from the $q^{+}=0$ frame (the standard choice within LFD, that always leads to $\left.q^{2} \leq 0\right)$ to a $q^{+} \neq 0$ frame, that allows both positive and negative $q^{2}$. Therefore, a covariant expression for the matrix elements of the EM current of hadrons is a useful tool for constructing viable approximations.

For instance, in the TL region the Mandelstam formula reads as follows

$$
j^{\mu}=-\imath e \int \frac{d^{4} k}{(2 \pi)^{4}} \operatorname{Tr}\left[S_{Q}\left(k_{Q}\right) \bar{\lambda}_{h}\left(q-k, k_{Q}, P_{h}\right) S(q-k) \Gamma^{\mu}(k, q) S(-k) \lambda_{\bar{h}}\left(k, k_{Q}, P_{\bar{h}}\right)\right]
$$

where $S(p)=1 /(\not p-m+\imath \epsilon)$, with $m$ the mass of the constituent struck by the virtual photon, $S_{Q}(p)$ is the propagator of the spectator constituent (e.g. a quark, or a diquark in a simple picture of baryons), $\lambda_{h}\left(k, k^{\prime}, P_{h}\right)$ is the hadron vertex function, which contains the Dirac structure, i.e. a proper combination of Dirac matrices, and a momentum dependence; $P_{h}^{\mu}\left(P_{\bar{h}}^{\mu}\right)$ is the hadron (antihadron) momentum; $\Gamma^{\mu}(k, q)$ is the quark-photon vertex, and $q^{\mu}$ is the virtual photon momentum. The corresponding SL expression can be obtained by applying the suitable changes to the hadron momenta and to the Dirac structure. The pion case is illustrated in detail in Refs. [3, 4]. It should be pointed out that the momentum components of both $\lambda_{h}$ and $\Gamma^{\mu}$ are necessary for regularizing Eq. (11).

Projecting out the Mandelstam formula on the Light-Front hyperplane, through a $k^{-}$ integration, allows us to introduce sensible approximations for constructing our phenomenological model. Let us remind that, presently, solving the Bethe-Salpeter equation for fermionic system represents a big challenge, and therefore phenomenological studies could play a very useful role.

Our assumptions for evaluating the $k^{-}$integration of Eq. (11) are: i) the momentum components of the vertex functions, both for the pion and for the vector mesons entering in our VMD model for $\Gamma^{\mu}(k, q)$, vanish in the $k^{-}$complex plane for $\left|k^{-}\right| \rightarrow \infty$, ii) the contributions of the vertex function singularities can be neglected. These approximations lead to the following issues: i) how to connect the Fock language with the Bethe-Salpeter one, e.g. how to describe both the amplitude for the emission/absorption of a pion by a 
quark (i.e. the nonvalence component of the pion wave function) and the $q \bar{q}$-pion vertex (i.e. the valence component), ii) how to model the quark-photon vertex. In the limit $m_{\pi} \rightarrow 0$, only the contribution generated by the nonvalence component of the hadron state, i.e. a higher Fock component, is acting in both TL and SL regions [ 3, 4], and therefore the analysis is remarkably simplified. In particular, in order to describe the emission/absorption of a pion by a quark, following Ref. [ 7] we assumed a pointlike interaction. It is worth noting that in the present approach, the coupling constant is fixed by the charge normalization of the pion form factor.

As to the quark-photon vertex, the bare term can be dropped, given our assumption on the pion mass, and one remains with the effect due to the $q \bar{q}$ production. In view of this, a VMD approximation has been applied, namely the relevant plus component of $\Gamma^{\mu}(k, q)$ is spanned over a VM basis as follows

$$
\Gamma^{+}(k, q)=\sqrt{2} \sum_{n, \lambda}\left[\epsilon_{\lambda} \cdot \widehat{V}_{n}\left(k, k-P_{n}\right)\right] \Lambda_{n}\left(k, P_{n}\right) \frac{\left[\epsilon_{\lambda}^{+}\right]^{*} f_{V n}}{\left(q^{2}-M_{n}^{2}+\imath M_{n} \tilde{\Gamma}_{n}\left(q^{2}\right)\right)}
$$

where $f_{V n}$ is the decay constant of the n-th vector meson into a virtual photon (to be calculated within our model), $M_{n}$ the corresponding mass, $\tilde{\Gamma}_{n}\left(q^{2}\right)$ the total decay width (equal to $\Gamma_{n} q^{2} / M_{n}^{2}$ for $q^{2}>0$ and vanishing for $q^{2} \leq 0$ ) and $\epsilon_{\lambda}$ the VM polarization. The VM vertex amplitude is $\left[\epsilon_{\lambda} \cdot \widehat{V}_{n}\left(k, k-P_{n}\right)\right] \Lambda_{n}\left(k, P_{n}\right)$, with a proper Dirac structure, $\widehat{V}_{n}\left(k, k-P_{n}\right)$, that generates the Melosh rotations for ${ }^{3} S_{1}$ states [8] and a momentumdependent part indicated by $\Lambda_{n}\left(k, P_{n}\right)$. In the kinematical region corresponding to the valence sector, the Bethe-Salpeter amplitudes, for both pion and vector mesons, are estimated through the eigenvectors of a relativistic simple model for pseudoscalar and vector mesons $[9]$.

An estimate of the probability of the valence component, $P_{q \bar{q}}$, is necessary for a proper normalization of such a component for both pion and vector mesons. In Ref. [3] a simple and intuitive model for $P_{q \bar{q}}$ is presented.

In the actual calculation, 20 vector mesons are taken into account to reach convergence up to $\left|q^{2}\right|=10(\mathrm{GeV} / \mathrm{c})^{2}$. The corresponding eigenstates are calculated from the model of Ref. [9], while for the masses a different choice has been made: for the first four vector mesons, the experimental values (together with the corresponding widths) have been considered, while for $M_{n}>2.150 \mathrm{GeV}$ the model eigenvalues have been adopted. We have introduced two adjusted parameters: i) a single width, $\Gamma_{n}$, for the vector mesons with mass $M_{n}>2.150 \mathrm{GeV}$, ii) the relative weight, $w_{V M}$, of the so-called "instantaneous" contributions of the pion and the vector mesons. These instantaneous terms are generated by the instantaneous part of the quark propagator, and they play the central role in the evaluation of the pion form factor, in the limit of a vanishing pion mass. We have chosen for the experimentally unknown widths a value $\Gamma_{n}=0.15 \mathrm{GeV}$, similar to the experimental width of the first four VM's; while for $w_{V M}$ we have found that $w_{V M}=-0.7$ is a good value for a global fit (see Fig. 1), and $w_{V M}=-1.5$ gives an improved description of the $\rho$ peak region (see Figs. 10 and 11 of [3]). 


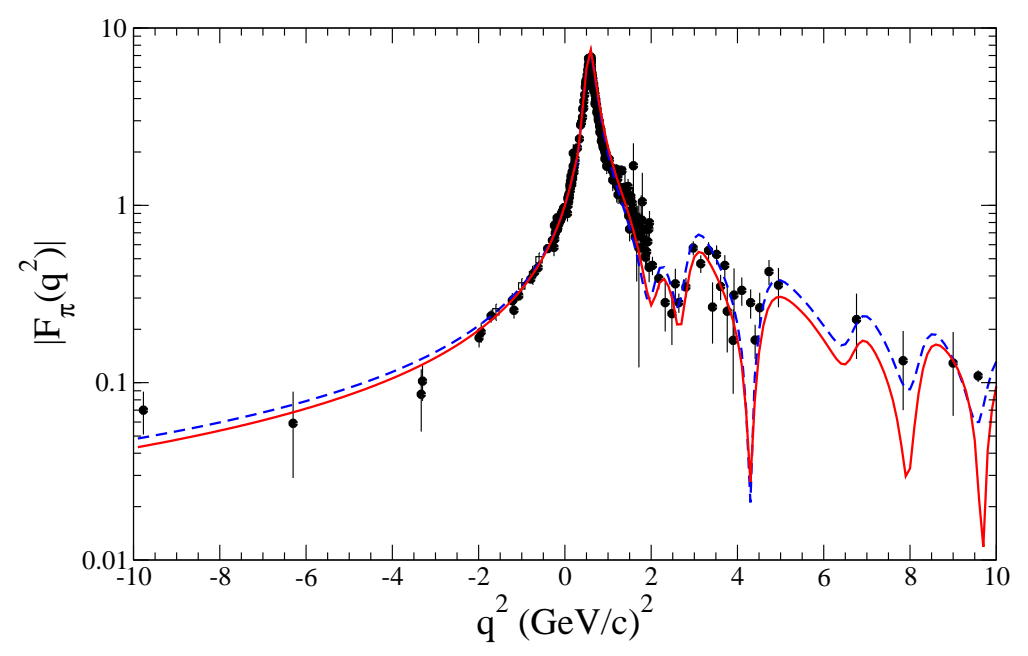

Figure 1. Pion EM form factor in the SL and TL regions vs $q^{2}$. Solid line: calculations with the pion wave function from the model of Ref. [9], and a relative weight $w_{V M}=$ -0.7 for the "instantaneous" terms. Dashed line: the same as the solid line, but with the asymptotic pion wave function [13]. Data from the collection of Ref. [10]. (After Ref. [3])

\section{The EM nucleon form factors}

The evaluation of the Sachs form factors of the nucleon largely proceeds along the same line of the pion. We consider the nucleon as a system of quarks with mass equal to $.220 \mathrm{GeV}$, and then we model the quark-nucleon amplitude in analogy with the pion case. First of all, we adopt a Dirac structure of the quark-nucleon vertex as suggested by an effective Lagrangian density like the one of Ref. [11], viz

$$
\begin{aligned}
& \mathcal{L}_{\text {eff }}\left(x, \tau_{1}, \tau_{2}, \tau_{3}, \tau_{N}\right)=\epsilon_{a b c} \int d^{4} x_{1} d^{4} x_{2} d^{4} x_{3} \mathcal{F}\left(x_{1}, x_{2}, x_{3}, x\right) \times \\
& {\left[m_{N} \alpha \frac{1}{\sqrt{2}} \sum_{\tau_{1}, \tau_{2}, \tau_{3}} \bar{q}^{a}\left(x_{1}\right) \mathcal{T}_{\tau_{1}}^{\dagger} \imath \tau_{y} \mathcal{T}_{\tau_{2}}^{*} \gamma^{5} q_{C}^{b}\left(x_{2}\right) \bar{q}^{c}\left(x_{3}\right) \mathcal{T}_{\tau_{3}}^{\dagger}+\right.} \\
& \left.-(1-\alpha) \frac{1}{\sqrt{6}} \sum_{\tau_{1}, \tau_{2}, \tau_{3}} \bar{q}^{a}\left(x_{1}\right) \mathcal{T}_{\tau_{1}}^{\dagger} \vec{\tau} \imath \tau_{y} \mathcal{T}_{\tau_{2}}^{*} \gamma^{5} \gamma_{\mu} q_{C}^{b}\left(x_{2}\right) \cdot \bar{q}^{c}\left(x_{3}\right) \mathcal{T}_{\tau_{3}}^{\dagger} \vec{\tau}\left(-\imath \partial^{\mu}\right)\right] \psi_{N}(x) \mathcal{T}_{\tau_{N}}+ \\
& \text { +cyclic }+ \text { h.c. }
\end{aligned}
$$

where $\mathcal{F}\left(x_{1}, x_{2}, x_{3}, x\right)$ is a scalar distribution that properly weights the quark coordinates; $\epsilon_{a b c}$ takes care of the color degrees of freedom; $m_{N}$ is the mass of the nucleon; $q(x, \tau)=$ $q(x) \mathcal{T}_{\tau}$ is the field that creates a quark $u$ or $d$ depending upon $\tau$; the charge conjugated field is $q_{C}(x, \tau)=\mathcal{C} q(x, \tau) \mathcal{C}^{\dagger}=C \bar{q}^{T}(x) \mathcal{T}_{\tau}^{C}=C \bar{q}^{T}(x) \imath \tau_{y} \mathcal{T}_{\tau}^{*}=\imath \gamma^{2} \gamma^{0} \bar{q}^{T}(x) \imath \tau_{y} \mathcal{T}_{\tau}^{*}$ and has a parity opposite to the quark field (this entails a pseudoscalar or an axial operator between $\bar{q}^{a}$ and $q_{C}^{b}$, in order to recover the correct intrinsic parity of the nucleon). The isospin field $\mathcal{T}_{\tau}^{C}=\imath \tau_{y} \mathcal{T}_{\tau}^{*}$ creates a charge conjugate isospin state, namely $\imath \tau_{y} \chi_{\tau_{2}}^{*}$. For the present time $\alpha=1$, i.e. we have considered a pseudoscalar coupling only.

In what follows, given the preliminary character of this presentation, we deal with the discussion of the EM form factor in the SL region. The matrix elements of the macroscopic EM current of the nucleon,

$$
\left\langle\sigma^{\prime}, p^{\prime}\left|j^{\mu}\right| p, \sigma\right\rangle=\bar{U}_{N}\left(p^{\prime}, \sigma^{\prime}\right)\left[-F_{2}\left(Q^{2}\right) \frac{p^{\prime \mu}+p^{\mu}}{2 M_{N}}+\left(F_{1}\left(Q^{2}\right)+F_{2}\left(Q^{2}\right)\right) \gamma^{\mu}\right] U_{N}(p, \sigma)
$$


where $Q^{2}=-q^{2}$ and $F_{1(2)}$ is the Dirac (Pauli) nucleon form factor, are approximated microscopically as follows

$$
\begin{aligned}
& \left\langle\sigma^{\prime}, p^{\prime}\left|j^{\mu}\right| p, \sigma\right\rangle=N_{c} \int \frac{d^{4} k_{1}}{(2 \pi)^{4}} \int \frac{d^{4} k_{2}}{(2 \pi)^{4}} \times \\
& \operatorname{Tr}\left\{\bar{\Phi}_{N}^{\sigma^{\prime}}\left(k_{1}, k_{2}, k_{3}^{\prime}, p^{\prime}\right) S^{-1}\left(k_{1}\right) S^{-1}\left(k_{2}\right) \mathcal{I}_{3}^{\mu} \Phi_{N}^{\sigma}\left(k_{1}, k_{2}, k_{3}, p\right)\right\}
\end{aligned}
$$

where the factor $N_{c}$ is a color weight, the trace is performed over isospin and Dirac matrices, $\Phi_{N}^{\sigma}\left(k_{1}, k_{2}, k_{3}, p\right)$ is the quark-nucleon Bethe-Salpeter amplitude [12], that contains both a Dirac structure as suggested by Eq. (3) and a momentum component, as in the pion case; $\mathcal{I}^{\mu}$ is the quark-photon vertex with isoscalar and isovector contributions, namely

$$
\mathcal{I}^{\mu}=\left(\frac{1+\tau_{z}}{2}\right) \mathcal{I}_{u}^{\mu}+\left(\frac{1-\tau_{z}}{2}\right) \mathcal{I}_{d}^{\mu}=\frac{\mathcal{I}_{u}^{\mu}+\mathcal{I}_{d}^{\mu}}{2}+\tau_{z} \frac{\mathcal{I}_{u}^{\mu}-\mathcal{I}_{d}^{\mu}}{2}=\mathcal{I}_{I S}^{\mu}+\tau_{z} \mathcal{I}_{I V}^{\mu}
$$

The terms $\mathcal{I}_{I S}^{\mu}$ and $\mathcal{I}_{I V}^{\mu}$ contain a pointlike (bare) contribution and a VMD contribution, with isoscalar $(\omega$-like) and isovector $(\rho$-like) content, respectively, viz

$$
\begin{aligned}
& \mathcal{I}_{I S(I V)}^{\mu}(k, q)=\mathcal{N}_{I S(I V)} \theta\left(p^{+}-k^{+}\right) \theta\left(k^{+}\right) \gamma^{\mu}+\theta\left(p^{+}-k^{+}\right) \theta\left(k^{+}-p^{+}\right) \times \\
& \left\{Z_{b} \mathcal{N}_{I S(I V)} \gamma^{\mu}+Z_{V} \Gamma_{V}^{\mu}[k, q, I S(I V)]\right\}
\end{aligned}
$$

where $\mathcal{N}_{I S}=1 / 6$ and $\mathcal{N}_{I S}=1 / 2$, the quantities $Z_{b}$ (bare term) and $Z_{V}$ (VMD term) are unknown renormalization constants to be extracted from the phenomenological analysis of the data. The theta functions, acting on the plus component of the momenta involved in the process, implement the kinematical constraints obtained from the projection of Eq. (5) on the LF hyperplane, as in the case of the pion. In particular, they identify the kinematical region relevant to the physical processes depicted in Fig. 2, that can be interpreted as valence and nonvalence contributions. Moreover, the vertical dashed lines in Fig. 2 allow one to count how many constituents are in flight, at a given LF time. It is worth noting that while the VMD is uniquely associated to the pair production by the virtual photon, (cf contribution $(b)$ in Fig. 2), i.e. to the nonvalence component of the nucleon, the bare term $\left(\gamma^{\mu}\right)$ can yield a contribution both in the elastic quark-photon channel (or triangle diagram, cf contribution (a) in Fig. 2) and in the pair production process.

The momentum dependence of the quark-nucleon amplitude in the valence sector, where the spectator quarks are on their-own $k^{-}$-shell, has been modeled adopting a power-law Ansatz [13], like

$$
\mathcal{W}_{N}\left(k_{1}, k_{3}, k_{3}, p\right) \sim \frac{1}{\left[\beta^{2}+M_{0}^{2}(1,2,3)\right]^{3}}
$$

where $\beta$ has been fixed through the anomalous magnetic moments of the nucleon. In particular, with $\beta=0.118 \mathrm{GeV}$, one obtains $\mu_{p}=2.878$ (Exp. 2.793) and $\mu_{n}=-1.859$ (Exp. -1.913), and $M_{0}(1,2,3)$ is the LF free mass for a three-quark system. In the non-valence sector, relevant for evaluating the Z-diagram contribution, the momentum dependence of the quark-nucleon amplitude is approximated by

$$
G_{N}\left(k_{1}, k_{3}, k_{3}, p\right) \sim \frac{1}{\left[\beta^{2}+M_{0}^{2}(1,2)\right]^{2}}\left\{\frac{1}{\left[\beta^{2}+M_{0}^{2}\left(3^{\prime}, 2\right)\right]}+\frac{1}{\left[\beta^{2}+M_{0}^{2}\left(3^{\prime}, 1\right)\right]}\right\}
$$




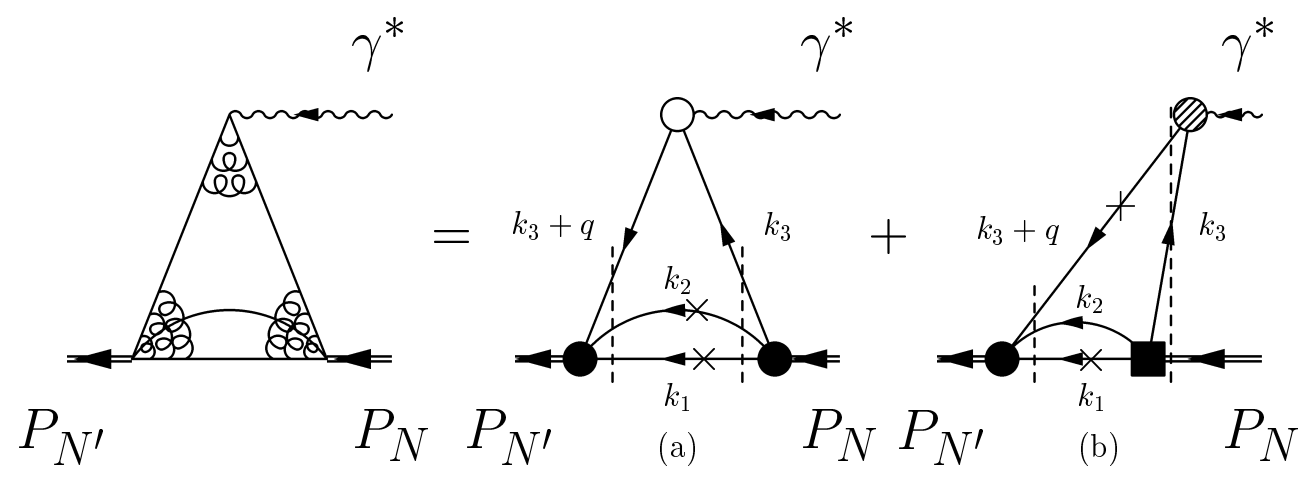

Figure 2. Diagrams contributing to the nucleon EM form factors in the spacelike region. Diagram $(a)$ (triangle or elastic term) illustrates the valence sector contribution, where $0<$ $k_{i}^{+}<P_{N}^{+}(i=1,2,3)$, and the symbol $\times$ on a quark line indicates that the corresponding quark is on its $k^{-}$-shell. Diagram $(b)$ shows the nonvalence sector contribution, $P_{N}^{+}<$ $k_{3}^{+}<P_{N}^{\prime+}$, due to the pair production.

where $M_{0}(i, j)$ is the LF free mass for a two-quark system. This Ansatz is different from the one used for the pion case, where a simple constant vertex was adopted, since the sixth-fold integration needs a stronger regularization.

For the present, preliminary calculations in the SL region we have the following adjusted parameters: i) $Z_{b}$ and $Z_{V}$, that are renormalization constants for the pair production terms in the quark-photon vertex (cf Eq. (7D) ), ii) two parameters for a running mass in the valence sector (with a range of variation of about $10 \%$, for $-10 \mathrm{GeV}^{2}<q^{2}<0$ ), iii) two parameters for a running mass in the non-valence sector (with a range of variation of about $20 \%$, for $\left.-10 \mathrm{GeV}^{2}<q^{2}<0\right)$.

The Sachs form factors can be obtained from the macroscopic current by applying the proper traces to Eq. (44) and then by using the microscopical evaluation of the relevant matrix elements. In particular in the frame where $\mathbf{p}_{\perp}^{\prime}=\mathbf{p}_{\perp}=0$ and $q^{+}=\sqrt{Q^{2}}$

$$
\begin{aligned}
& G_{E}^{N}\left(Q^{2}\right)=F_{1}\left(Q^{2}\right)-\frac{Q^{2}}{4 M_{N}^{2}} F_{2}\left(Q^{2}\right)= \\
& =\frac{M_{N}^{2}}{2 p^{+} p^{\prime+}} \operatorname{Tr}\left\{\frac{\not p^{\prime}+M_{N}}{2 M_{N}}\left[-F_{2}^{N}\left(Q^{2}\right) \frac{p^{\prime+}+p^{+}}{2 M_{N}}+\left(F_{1}^{N}\left(Q^{2}\right)+F_{2}^{N}\left(Q^{2}\right)\right) \gamma^{+}\right] \frac{\not p+M_{N}}{2 M_{N}} \gamma^{+}\right\} \\
& G_{M}^{N}\left(Q^{2}\right)=F_{1}\left(Q^{2}\right)+F_{2}\left(Q^{2}\right)= \\
& =\frac{2 M_{N}^{2}}{Q^{2}} \operatorname{Tr}\left\{\frac{\not p^{\prime}+M_{N}}{2 M_{N}}\left[-F_{2}^{N}\left(Q^{2}\right) \frac{p^{\prime}+p^{1}}{2 M_{N}}+\left(F_{1}^{N}\left(Q^{2}\right)+F_{2}^{N}\left(Q^{2}\right)\right) \gamma^{1}\right] \frac{\not p+M_{N}}{2 M_{N}} \gamma^{1}\right\}(10)
\end{aligned}
$$

In Figs. 3 and 4 , the ratio $G_{E}^{p}\left(Q^{2}\right) \mu_{p} / G_{M}^{p}\left(Q^{2}\right)$, and the form factors $G_{M}^{p}\left(Q^{2}\right), G_{E}^{n}\left(Q^{2}\right)$, $G_{M}^{n}\left(Q^{2}\right)$ are shown. One of the main feature of our preliminary calculation is the explanation of the possible zero in the ratio $G_{E}^{p}\left(Q^{2}\right) \mu_{p} / G_{M}^{p}\left(Q^{2}\right)$ in terms of the interplay between the Z-diagram contribution, i.e. higher Fock components, and the elastic contribution. Such an interpretation appears supported by the encouraging agreement between theoretical calculations and experimental data. 

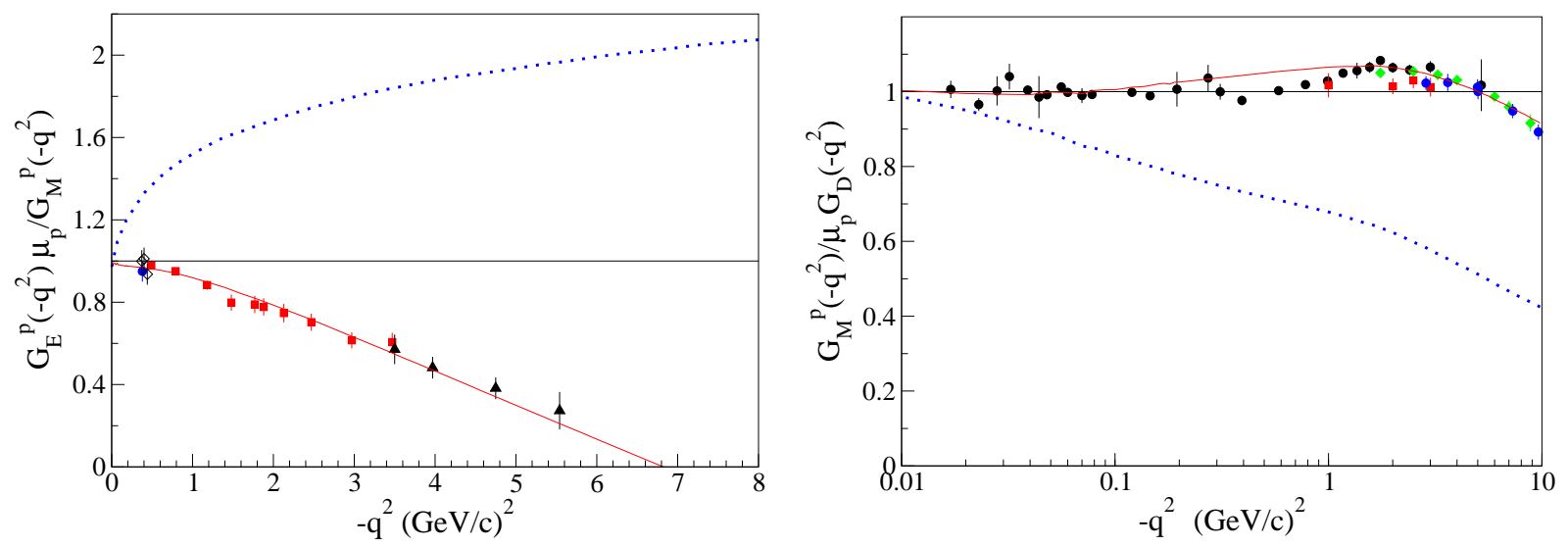

Figure 3. Left panel. The ratio $G_{E}^{p}\left(Q^{2}\right) \mu_{p} / G_{M}^{p}\left(Q^{2}\right)$ vs $Q^{2}$. Solid line: full calculation, corresponding to the sum of all the contributions to $G_{E}^{p}\left(Q^{2}\right)$ and $G_{M}^{p}\left(Q^{2}\right)$, i.e. triangle plus pair production terms. Dotted line: triangle elastic contribution to $G_{E}^{p}\left(Q^{2}\right)$ and $G_{M}^{p}\left(Q^{2}\right)$. Data: from [14]. Right panel. $G_{M}^{p}\left(Q^{2}\right) / \mu_{p} G_{D}\left(Q^{2}\right)$ vs $Q^{2}$, with the same notations as in the left panel, and $G_{D}\left(Q^{2}\right)=1 /\left(1+Q^{2} / 0.71\right)^{2}$.
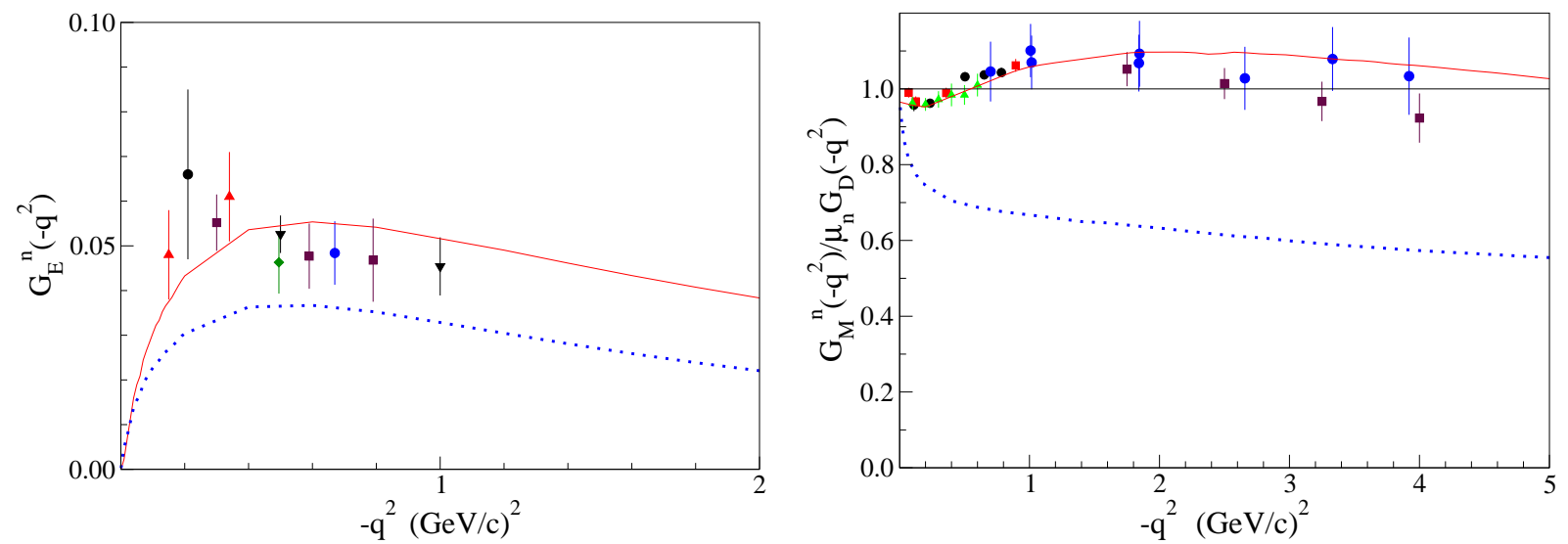

Figure 4. Left panel. $G_{E}^{n}\left(Q^{2}\right)$ vs $Q^{2}$. Solid line: full calculation, corresponding to the sum of all the contributions to $G_{E}^{n}\left(Q^{2}\right)$, i.e. triangle plus pair production terms. Dotted line: triangle elastic contribution to $G_{E}^{n}\left(Q^{2}\right)$. Data: from [ 14]. Right panel. $G_{M}^{n}\left(Q^{2}\right) / \mu_{n} G_{D}\left(Q^{2}\right)$ vs $Q^{2}$, with the same notations as in the left panel, and $G_{D}\left(Q^{2}\right)=$ $1 /\left(1+Q^{2} / 0.71\right)^{2}$. 


\section{Conclusions \& Perspectives}

In [ 3, 4], we constructed a microscopical model for the pion form factor, within a LF approach, obtaining a very nice description of experimental data. The development of this model for calculating EM nucleon form factors in both SL and TL regions is our next goal. Our model for the nucleon exploits many ingredients already introduced for the pion, adding suitable Ansatzes for the quark-nucleon amplitude in both the valence and nonvalence sectors. The possibility to address separately valence and nonvalence sectors of the hadron amplitude represents a distinct feature of our phenomenological model, and it is entailed by the model for the quark-photon vertex, we have adopted. In particular, within our approach one could ascribe the possible zero in the ratio $G_{E}^{p}\left(Q^{2}\right) \mu_{p} / G_{M}^{p}\left(Q^{2}\right)$ to the interplay between the contribution from the pair production process and the one from the elastic quark-photon vertex, shedding some light on the deep meaning of the puzzling experimental finding.

The next step will be the extension of our calculation to the timelike region.

\section{REFERENCES}

1. P. A. M. Dirac, Rev. Mod. Phys. 21 (1949) 392.

2. S. J. Brodsky, H. C. Pauli, and S. S. Pinsky, Phys. Rep. 301 (1998) 299.

3. J. P. B. C. de Melo, T. Frederico, E. Pace and G. Salmè, Phys. Rev. D 73 (2006) 074013.

4. J. P. B. C. de Melo, T. Frederico, E. Pace and G. Salmè, Phys. Lett. B 581 (2004) 75.

5. F. M. Lev, E. Pace and G. Salmè, Nucl. Phys. A 641 (1998) 229; Phys. Rev. Lett. 83 (1999) 5250.

6. S. Mandelstam, Proc. Royal Soc. (London) A 233 (1956) 248.

7. C.-R. Ji and H.-M. Choi, Phys. Lett. B 513 (2001) 330.

8. W. Jaus, Phys. Rev. D 41 (1990) 3394.

9. T. Frederico, H.-C. Pauli and S.-G. Zhou, Phys. Rev. D 66 (2002) 054007; Phys. Rev. D 66 (2002) 116011.

10. R. Baldini, et al., Eur. Phys. J. C 11, 709 (1999); Nucl. Phys. A 666 \& 667 (2000) 3 ; and private communication.

11. W. R. de Araujo, E. F. Suisso, T. Frederico, M. Beyer and H. J. Weber, Phys. Lett. B 478 (2000) 86.

12. J. P. B. C. de Melo, T. Frederico, E. Pace, S. Pisano and G. Salmè, to be published.

13. G. P. Lepage and S. J. Brodsky, Phys. Rev. D 222157 (1980) 2157.

14. www.jlab.org/ cseely/nucleons.html and Refs. therein. 